\title{
Research Article \\ One-Dimensional Problem of a Conducting Viscous Fluid with One Relaxation Time
}

\author{
Angail A. Samaan \\ Department of Mathematics, Faculty of Girls, Ain Shams University, Cairo 11757, Egypt \\ Correspondence should be addressed to Angail A. Samaan, angail123@yahoo.com
}

Received 2 August 2010; Revised 28 December 2010; Accepted 23 February 2011

Academic Editor: Sergio Preidikman

Copyright (C 2011 Angail A. Samaan. This is an open access article distributed under the Creative Commons Attribution License, which permits unrestricted use, distribution, and reproduction in any medium, provided the original work is properly cited.

We introduce a magnetohydrodynamic model of boundary-layer equations for conducting viscous fluids. This model is applied to study the effects of free convection currents with thermal relaxation time on the flow of a viscous conducting fluid. The method of the matrix exponential formulation for these equations is introduced. The resulting formulation together with the Laplace transform technique is applied to a variety problems. The effects of a plane distribution of heat sources on the whole and semispace are studied. Numerical results are given and illustrated graphically for the problem.

\section{Introduction}

The modification of the heat-conduction equation from diffusive to a wave type may be affected either by a microscopic consideration of the phenomenon of heat transport or in a phenomenological way by modifying the classical Fourier law of heat conduction.

Many authors have considered various aspects of this problem and obtained similarity solutions. Samaan [1] investigated steady oscillating magnetohydrodynamic flow in a circular pipe. Analytical and numerical methods for the momentum and energy equations of a viscous incompressible fluid along a vertical plate have been considered by Samaan [2]. Chamkha [3] studied the magnetohydrodynamic flow of a uniformly stretched vertical permeable surface in the presence of heat generation/absorption and chemical reaction. Ishak et al. [4] investigated theoretically the unsteady mixed convection boundary layer flow and heat transfer due to a stretching vertical surface in a quiescent viscous and incompressible fluid.

Many authors presented some mathematical results, and a good amount of references can be found in the papers by Liao and Pop [5] and Nazar et al. [6]. Further, the stagnation region encounters the highest pressure, the highest heat transfer, and the highest rate of mass 
deposition studied by Wang [7]. Singh et al. [8] investigated the problem of heat transfer in the flow of an incompressible fluid.

Samaan [9] investigated the heat and mass transfer over an accelerating surface with heat source in presence of suction and magnetic field. The flow of an unsteady, incompressible magnetohydrodynamics (MHDs) viscous fluid with suction is investigated by Muhammad et al. [10]. Heat and mass transfer over an accelerating surface with heat source in presence of magnetic field is derived by Samaan [11].

Recently, Samaan [12] studied the effects of variable viscosity and thermal diffusivity on the steady flow in the presence of the magnetic field. variable viscosity effects on hydrodynamic boundary layer flow along a continuously moving vertical plate were done by Mostafa [13]. Concerning the studies of state space formulation for MHDs and free convection flow with two relaxation times and the free convection effective perfectly conducting couple stress fluid, we may refer to Samaan [14], Ezzat et al. [15], and Hayat et al. [16]. Using differential transform method and Pade approximate for solving MHDs flow in a laminar liquid film from a horizontal stretching surfaces investigated by Rashidi et al. [17].

In the present work, we use a more general model of magnetohydrodynamic free convection flow, which also includes the relaxation time of heat conduction and the electric displacement current $[18,19]$. An attempt to account for the time dependence of heat transfer, Cattaneo [20] and Vernotte [21] independently modified Fourier's law to include the relaxation time of the system. Generalized thermoelasticity stands for a hyperbolic thermoelasticity in which a thermomechanical load applied to a body is transmitted in a wave-like manner throughout the body, only transient thermoelastic waves are included in the survey by Hertnarski and Ignaczak [22]. The inclusion of the relaxation time and the electric displacement current modifies the governing thermal and electromagnetic field equations, changing them from the parabolic to a hyperbolic type, and thereby eliminating the unrealistic result that thermal and electromagnetic disturbances are realized instantaneously within a fluid.

The solution is obtained using a state-space approach. The importance of state-space analysis is recognized in fields where the time behavior of physical process is of interest. The first writer to introduce the state space approach in magnetohydrodynamic free convection flow was Ezzat $[23,24]$. His works dealt with free convection flow in the absence of the applied magnetic field or when there are no heat sources. The present work is an attempt to generalize these results to include the effects of heat sources. The results obtained are used to solve a problem for the whole space with a plane distribution of heat sources. The solutions obtained are utilized in combination with the method of images to obtain the solution for a problem with heat sources distributed situated inside a semispace whose surface bounded by an infinite vertical plate.

The Laplace transform techniques is applied to one-dimensional problem. The inversion of the Laplace transforms is carried out using a numerical technique [25].

\section{Formulation of the Problem}

Let a constant magnetic field of strength $H_{0}$ act in the direction of the $y$-axis. Due to the effect

of this magnetic field, there arises in the medium an induced magnetic field $h$ and an induced electric field $\underset{\rightarrow}{E}$. All the considered functions will depend on $y$ and the time $\vec{t}$ only. 
The electromagnetic quantities satisfy Maxwell's equations [26]

$$
\begin{gathered}
\underset{\rightarrow}{\operatorname{curl}} \underset{\rightarrow}{h}=J+\varepsilon_{0} \dot{E}, \\
\operatorname{curl} \underset{\rightarrow}{E}=-\mu_{0} \dot{h}, \\
\operatorname{div} \underset{\rightarrow}{h}=0, \quad \operatorname{div} \underset{\rightarrow}{E}=0, \\
\underset{\rightarrow}{\rightarrow}=\mu_{o}\left(\underset{\rightarrow}{H_{0}}+\underset{\rightarrow}{h}\right), \quad \underset{\rightarrow}{D}=\varepsilon_{0} \underset{\rightarrow}{E},
\end{gathered}
$$

where $J$ is the electric current density, $\mu_{o}$ and $\varepsilon_{o}$ are the magnetic and electric permeabilities, respectively, and $\underset{B}{\vec{D}} \rightarrow \overrightarrow{\mathrm{D}}$ are the magnetic and electric induction vectors, respectively, and dotte is the time variable.

These equations are supplemented by Ohm's law

$$
\underset{\rightarrow}{J}=\sigma_{o}\left[\underset{\rightarrow}{E}+\mu_{o}\left(\underset{\rightarrow}{v} \times \underset{\rightarrow}{H_{0}}\right)\right]
$$

where $\underset{\vec{v}}{v}=(u, 0,0)$ is velocity vector of the fluid and $\sigma_{o}$ is the electric conductivity.

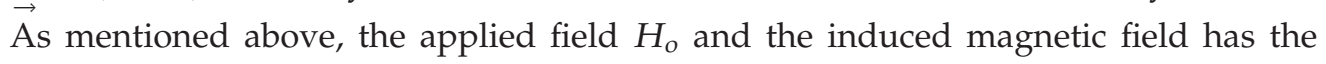
components

$$
\underset{\rightarrow}{H_{0}}=\left(0, H_{0}, 0\right), \quad \stackrel{h}{\rightarrow}=(h, 0,0) .
$$

The vector $\underset{\rightarrow}{E}$ and $\underset{\rightarrow}{J}$ will have nonvanishing components only in the $z$-direction. That is,

$$
\underset{\rightarrow}{E}=(0,0, E), \quad \underset{\rightarrow}{J}=(0,0, J),
$$

where from (2.5)

$$
J=\sigma_{o}\left[E+\mu_{o} H_{o} u\right]
$$

The vector (2.1) and (2.2) reduce to the following scalar equations

$$
\begin{gathered}
h_{, y}=-\left(J+\varepsilon_{o} \dot{E}\right), \\
E_{, y}=-\mu_{o} \dot{h} .
\end{gathered}
$$

Eliminating $J$ between (2.8) and (2.9), we obtain

$$
h_{, y}=-\left(\sigma_{o} E+\varepsilon_{o} \dot{E}\right)-\sigma_{o} \mu_{o} H_{o} u
$$


The pondermotive force $\underset{\rightarrow}{F}=\underset{\rightarrow}{J} \times \underset{\rightarrow}{B}$ has one nonvanishing component in the $x$-direction

$$
F_{x}=\mu_{o} H_{o}\left(h_{, y}+\varepsilon_{o} \dot{E}\right)
$$

where $\sigma_{o}$ is the electric conductivity.

We investigate the free convective heat transfer in an incompressible hydromagnetic flow past an infinite vertical plate. The $x$-axis is taken along the plate in the direction of the flow and the $y$-axis normal to it. Let $u$ be the component of the velocity in the $x$ direction. All the fluid properties are assumed constant, except that the influence of the density variation with temperature is considered only in the body force term. In the energy equations, terms representing viscous and Joule's dissipation are neglected, as they are assumed to be very small in free convection flow [27]. Also, in the energy equation, the term representing the volumetric heat source is taken as a function of the space variables. In view of the assumptions, the equations that govern unsteady one-dimensional free convection flow in an incompressible conducting fluid through a porous medium bounded by an infinite nonmagnetic vertical plate in the presence of a constant magnetic field are (2.10)-(2.12), and the equations describing the flow in the boundary layer reduce to [28-30]

$$
\begin{gathered}
\rho \dot{u}=\rho v u_{, y y}-\rho \frac{v}{K} u+\rho \frac{\alpha^{2}}{H_{o}}\left(h_{, y}+\varepsilon_{o} \dot{E}\right)+\rho g \beta\left(T-T_{\infty}\right), \\
h_{, y}=-\left(\sigma_{o} E+\varepsilon_{o} \dot{E}\right)-\sigma_{o} \mu_{o} H_{o} u, \\
E_{, y}=-\mu_{o} \dot{h}, \\
\rho c_{p} \dot{T}=\lambda T_{, y y}-\rho c_{p} v_{o} \ddot{T}+Q v_{o} \dot{\mathcal{Q}} .
\end{gathered}
$$

And the constitutive equation

$$
\left(\rho_{\infty}-\rho\right)=\rho \beta\left(T-T_{\infty}\right)
$$

In these equations, $K$ is the permeability of the porous medium, $\alpha$ is the Alfven velocity, $g$ is the acceleration due to gravity, $\beta$ is the coefficient of volume expansion, $T$ is the temperature distribution, $T_{\infty}$ is the temperature of the fluid away from the plate, $c_{p}$ is specific heat at constant pressure, $\lambda$ is the thermal diffusivity, $v_{o}$ is the relaxation time, $\rho_{\infty}$ is the density of the fluid far from the surface, and $\rho$ is the density of the fluid.

Let us introduce the following nondimensional variables

$$
\begin{aligned}
& y^{*}=\frac{y \alpha}{v}, \quad t^{*}=\frac{t \alpha^{2}}{v}, \quad u^{*}=\frac{u}{\alpha}, \quad E^{*}=\frac{E}{\mu_{o} H_{o} \alpha}, \quad h^{*}=\frac{h}{H_{o}}, \quad v_{o}^{*}=\frac{\alpha^{2} v_{o}}{v}, \\
& K^{*}=\frac{K \alpha^{2}}{v^{2}}, \quad \theta=\frac{T-T_{\infty}}{T_{o}-T_{\infty}}, \quad G_{r}=\frac{v \beta g\left(T_{o}-T_{\infty}\right)}{\alpha^{3}}, \quad Q^{*}=\frac{v^{2} Q}{\lambda \alpha^{2}\left(T_{o}-T_{\infty}\right)} .
\end{aligned}
$$


In view of these transformations, (2.13)-(2.14) become

$$
\begin{gathered}
\dot{u}=u_{, y y}-\frac{1}{K} u+h_{, y}+a \dot{E}+G_{r} \theta, \\
h_{, y}=-\eta(E+u)-a \dot{E}, \\
E_{y}=-\dot{h}, \\
\theta_{, y y}-p_{r}\left(\dot{\theta}+v_{o} \ddot{\theta}\right)=-Q-v_{o} \dot{\mathcal{Q}},
\end{gathered}
$$

where $G_{r}$ is the Grashof number, $c$ is the speed of light given by $c^{2}=1 / \varepsilon_{0} \mu_{o}, a=\alpha^{2} / c^{2}$, $\eta=v \mu_{o} \sigma_{o}$ is a measure of the magnetic viscosity, and $p_{r}=\rho c_{p} v / \lambda$ is the Prandtl number. From now on, we will consider a heat source of the form

$$
Q=Q_{o} \delta(y) H(t)
$$

where $\delta(y)$ and $H(t)$ are the Dirac delta function and the Heaviside unit step function, respectively, $Q$ is the strength of the applied heat source, and $Q_{o}$ is a constant.

We will assume that the initial state of the medium is quiescent. Taking the Laplace transform, defined by the relation

$$
\bar{f}(s)=\int_{0}^{\infty} e^{-s t} f(t) d t
$$

of both sides of (2.16), we obtain

$$
\begin{gathered}
\frac{\partial^{2} \bar{u}}{\partial y^{2}}-\left(\frac{1}{K}+s\right) \bar{u}=-G_{r} \bar{\theta}-\frac{\partial \bar{h}}{\partial y}-a s \bar{E} \\
\frac{\partial \bar{h}}{\partial y}=-(\eta+a s) \bar{E}-\eta \bar{u} \\
\frac{\partial \bar{E}}{\partial y}=-s \bar{h} \\
{\left[\frac{\partial^{2}}{\partial y^{2}}-p_{r} s\left(1+\tau_{o} s\right)\right] \theta=-Q_{o} \frac{\left(1+\tau_{o} s\right)}{s} \delta(y) .}
\end{gathered}
$$

Eliminating $E$ between (2.19)-(2.20), we get

$$
\begin{gathered}
\left(\frac{\partial^{2}}{\partial y^{2}}-F\right) \bar{u}=-G_{r} \bar{\theta}-n \frac{\partial \bar{h}}{\partial y} \\
\left(\frac{\partial^{2}}{\partial y^{2}}-\xi\right) \bar{h}=-\eta \frac{\partial u}{\partial y}
\end{gathered}
$$


where

$$
F=s+\frac{1}{K}+\frac{a s \eta}{\eta+a s}, \quad n=\frac{\eta}{\eta+a s}, \quad \xi=s(\eta+a s)
$$

We will choose as state variables the temperature $\theta$, the velocity component $u$, and induced magnetic field $h$ and their gradients. Equations (2.22)-(2.23) can be written as

$$
\begin{gathered}
\frac{\partial \bar{\theta}}{\partial y}=\bar{\theta}^{\prime}, \quad \frac{\partial \bar{u}}{\partial y}=\bar{u}^{\prime}, \quad \frac{\partial \bar{h}}{\partial y}=\bar{h}^{\prime}, \\
\frac{\partial \bar{\theta}^{\prime}}{\partial y}=p s \bar{\theta}-Q_{o} \delta(y)\left(\frac{1+v_{o} s}{s}\right), \\
\frac{\partial \bar{u}^{\prime}}{\partial y}=-G_{r} \bar{\theta}+F \bar{u}-n \bar{h}^{\prime}, \\
\frac{\partial \bar{h}^{\prime}}{\partial y}=-\eta \bar{u}^{\prime}+\xi \bar{h},
\end{gathered}
$$

where $p=p_{r}\left(1+v_{o} s\right)$. The above equations can be written in matrix form as

$$
\frac{d \bar{v}(y, s)}{d y}=A(s) \bar{v}(y, s)+B(y, s)
$$

where

$$
A(s)=\left[\begin{array}{cccccc}
0 & 0 & 0 & 1 & 0 & 0 \\
0 & 0 & 0 & 0 & 1 & 0 \\
0 & 0 & 0 & 0 & 0 & 1 \\
p s & 0 & 0 & 0 & 0 & 0 \\
-G_{r} & F & 0 & 0 & 0 & -n \\
0 & 0 & \xi & 0 & -\eta & 0
\end{array}\right], \quad \bar{v}(y, s)=\left[\begin{array}{c}
\bar{\theta}(y, s) \\
\bar{u}(y, s) \\
\bar{h}(y, s) \\
\bar{\theta}^{\prime}(y, s) \\
\bar{u}^{\prime}(y, s) \\
\bar{h}^{\prime}(y, s)
\end{array}\right],
$$

$$
B(y, s)=-Q_{o} \delta(y)\left(\frac{1+v_{o} S}{s}\right)\left[\begin{array}{l}
0 \\
0 \\
0 \\
1 \\
0 \\
0
\end{array}\right] .
$$

In order to solve the system (2.26), we need first to find the form of the matrix $\exp (A(s) y)$. 
The characteristic equation of the matrix $A$ has the form

$$
k^{6}-Z_{1} k^{4}+Z_{2} k^{2}-Z_{3}=0
$$

where

$$
\begin{gathered}
Z_{1}=F+\xi+p s+\eta n, \\
Z_{2}=F \xi+p s(F+\xi)+\eta n p s, \\
Z_{3}=p s F \xi .
\end{gathered}
$$

The roots $\pm k_{1}, \pm k_{2}$, and $\pm k_{3}$ of (2.28) satisfy the relations

$$
\begin{gathered}
Z_{1}=k_{1}^{2}+k_{2}^{2}+k_{3}^{2}, \\
Z_{2}=k_{1}^{2} k_{2}^{2}+k_{2}^{2} k_{3}^{2}+k_{1}^{2} k_{3}^{2}, \\
Z_{3}=k_{1}^{2} k_{2}^{2} k_{3}^{2} .
\end{gathered}
$$

One of the roots, say $k_{1}^{2}$, has a simple expression given by

$$
k_{1}^{2}=p s
$$

The other two roots $k_{2}^{2}$ and $k_{3}^{2}$ satisfy the relation

$$
\begin{gathered}
k_{2}^{2}+k_{3}^{2}=F+\xi+\eta n, \\
k_{2}^{2} k_{3}^{2}=F \xi .
\end{gathered}
$$

The Taylor series expansion of the matrix exponential has the form

$$
\exp [A(s) y]=\sum_{n=0}^{\infty} \frac{1}{n !}[A(s) y]^{n}
$$

Using the well-known Cayley-Hamilton theorem, the infinite series can be truncated to the following form:

$$
\exp [A(s) y]=L(s, y)=b_{o} I+b_{1} A+b_{2} A^{2}+b_{3} A^{3}+b_{4} A^{4}+b_{5} A^{5}
$$


where $I$ is the unit matrix of order 6 and $b_{0}-b_{5}$ are some parameters depending on $s$ and $y$. The characteristic roots $\pm k_{i}, i=1,2,3$ of the matrix $A$ must satisfy the equations

$$
\begin{aligned}
& \exp \left( \pm k_{1} y\right)=b_{o} \pm b_{1} k_{1}+b_{2} k_{1}^{2} \pm b_{3} k_{1}^{3}+b_{4} k_{1}^{4} \pm b_{5} k_{1}^{5} \\
& \exp \left( \pm k_{2} y\right)=b_{o} \pm b_{1} k_{2}+b_{2} k_{2}^{2} \pm b_{3} k_{2}^{3}+b_{4} k_{2}^{4} \pm b_{5} k_{2}^{5} \\
& \exp \left( \pm k_{3} y\right)=b_{o} \pm b_{1} k_{3}+b a_{2} k_{3}^{2} \pm b_{3} k_{3}^{3}+b_{4} k_{3}^{4} \pm b_{5} k_{3}^{5}
\end{aligned}
$$

The solution of this system of linear equations is given by

$$
\begin{gathered}
b_{o}=-R\left(k_{2}^{2} k_{3}^{2} C_{1}+k_{1}^{2} k_{3}^{2} C_{2}+k_{2}^{2} k_{1}^{2} C_{3}\right), \\
b_{1}=-R\left(k_{2}^{2} k_{3}^{2} S_{1}+k_{3}^{2} k_{1}^{2} S_{2}+k_{1}^{2} k_{2}^{2}\right), \\
b_{2}=R\left[\left(k_{2}^{2}+k_{3}^{2}\right) C_{1}+\left(k_{3}^{2}+k_{1}^{2}\right) C_{2}+\left(k_{1}^{2}+k_{2}^{2}\right) C_{3}\right], \\
b_{3}=R\left[\left(k_{2}^{2}+k_{3}^{2}\right) S_{1}+\left(k_{3}^{2}+k_{1}^{2}\right) S_{2}+\left(k_{1}^{2}+k_{2}^{2}\right) S_{3}\right], \\
b_{4}=-R\left(C_{1}+C_{2}+C_{3}\right), \\
b_{5}=-R\left(S_{1}+S_{2}+S_{3}\right),
\end{gathered}
$$

where

$$
\begin{gathered}
R=\frac{1}{\left(k_{1}^{2}-k_{2}^{2}\right)\left(k_{2}^{2}-k_{3}^{2}\right)\left(k_{3}^{2}-k_{1}^{2}\right)}, \\
C_{1}=\left(k_{2}^{2}-k_{3}^{2}\right) \cosh \left(k_{1} y\right), \quad S_{1}=\frac{\left(k_{2}^{2}-k_{3}^{2}\right)}{k_{1}} \sinh \left(k_{1} y\right), \\
C_{2}=\left(k_{3}^{2}-k_{1}^{2}\right) \cosh \left(k_{2} y\right), \quad S_{2}=\frac{\left(k_{3}^{2}-k_{1}^{2}\right)}{k_{2}} \sinh \left(k_{2} y\right), \\
C_{3}=\left(k_{1}^{2}-k_{2}^{2}\right) \cosh \left(k_{3} y\right), \quad S_{3}=\frac{\left(k_{1}^{2}-k_{2}^{2}\right)}{k_{3}} \sinh \left(k_{3} y\right) .
\end{gathered}
$$

Substituting for the parameters $b_{0}-b_{5}$ from (2.36) into (2.34) and computing $A^{2}, A^{3}, A^{4}$, and $A^{5}$, we get the elements $\left(L_{i j}=1,2,3,4,5,6\right)$ of the matrix $L(s, y)$ to be

$$
\begin{aligned}
& L_{11}=R\left(k_{1}^{2}-k_{2}^{2}\right)\left(k_{3}^{2}-k_{1}^{2}\right) C_{1}, \\
& L_{12}=L_{13}=0,
\end{aligned}
$$




$$
\begin{aligned}
& L_{14}=R\left(k_{1}^{2}-k_{2}^{2}\right)\left(k_{3}^{2}-k_{1}^{2}\right) S_{1}, \\
& L_{15}=L_{16}=0 \text {, } \\
& L_{21}=G_{r} R\left[\left(k_{1}^{2}-\xi\right) C_{1}+\left(k_{2}^{2}-\xi\right) C_{2}+\left(k_{3}^{2}-\xi\right) C_{3}\right], \\
& L_{22}=R\left[\left(k_{1}^{2}-k_{2}^{2}\right)\left(F-k_{3}^{2}\right) C_{2}+\left(k_{1}^{2}-k_{3}^{2}\right)\left(F-k_{2}^{2}\right) C_{3}\right] \text {, } \\
& L_{23}=n \xi R\left[\left(k_{2}^{2}-k_{1}^{2}\right) S_{2}+\left(k_{3}^{2}-k_{1}^{2}\right) S_{3}\right], \\
& L_{24}=G_{r} R\left[\left(k_{1}^{2}-\xi\right) S_{1}+\left(k_{2}^{2}-\xi\right) S_{2}+\left(k_{3}^{2}-\xi\right) S_{3}\right], \\
& L_{25}=R\left[\left(k_{2}^{2}-\xi\right)\left(k_{1}^{2}-k_{2}^{2}\right) S_{2}+\left(k_{3}^{2}-\xi\right)\left(k_{1}^{2}-k_{3}^{2}\right) S_{3}\right], \\
& L_{26}=n R\left[\left(k_{2}^{2}-k_{1}^{2}\right) C_{2}+\left(k_{3}^{2}-k_{1}^{2}\right) C_{3}\right] \text {, } \\
& L_{31}=-G_{r} \eta R\left[k_{1}^{2} S_{1}+k_{2}^{2} S_{2}+k_{3}^{2} S_{3}\right], \\
& L_{32}=\eta F R\left[\left(k_{2}^{2}-k_{1}^{2}\right) S_{2}+\left(k_{3}^{2}-k_{1}^{2}\right) S_{3}\right], \\
& L_{33}=R\left[\left(n-k_{3}^{2}\right)\left(k_{1}^{2}-k_{2}^{2}\right) C_{2}+\left(n-k_{2}^{2}\right)\left(k_{1}^{2}-k_{3}^{2}\right) C_{3}\right], \\
& L_{34}=-G_{r} \eta R\left[C_{1}+C_{2}+C_{3}\right] \text {, } \\
& L_{35}=\eta R\left[\left(k_{2}^{2}-k_{1}^{2}\right) C_{2}+\left(k_{3}^{2}-k_{1}^{2}\right) C_{3}\right] \text {, } \\
& L_{36}=R\left[\left(k_{2}^{2}-F\right)\left(k_{1}^{2}-k_{2}^{2}\right) S_{2}+\left(k_{3}^{2}-F\right)\left(k_{1}^{2}-k_{3}^{2}\right) S_{3}\right] \text {, } \\
& L_{41}=-R k_{1}^{2}\left(k_{1}^{2}-k_{2}^{2}\right)\left(k_{1}^{2}-k_{3}^{2}\right) S_{1}, \\
& L_{42}=L_{43}=0 \text {, } \\
& L_{44}=-R\left(k_{1}^{2}-k_{2}^{2}\right)\left(k_{1}^{2}-k_{3}^{2}\right) C_{1}, \\
& L_{45}=L_{46}=0 \text {, } \\
& L_{51}=G R\left[k_{1}^{2}\left(k_{1}^{2}-\xi\right) S_{1}+k_{2}^{2}\left(k_{2}^{2}-\xi\right) S_{2}+k_{3}^{2}\left(k_{3}^{2}-\xi\right) S_{3}\right], \\
& L_{52}=F R\left[\left(k_{1}^{2}-k_{2}^{2}\right)\left(k_{1}^{2}-\xi\right) S_{1}+\left(k_{1}^{2}-k_{3}^{2}\right)\left(k_{3}^{2}-\xi\right) S_{3}\right], \\
& L_{53}=\frac{n \xi R}{\eta} L_{35} \\
& L_{54}=L_{21} \text {, } \\
& L_{55}=R\left[\left(k_{1}^{2}-k_{2}^{2}\right)\left(k_{2}^{2}-n\right) C_{2}+\left(k_{1}^{2}-k_{3}^{2}\right)\left(k_{3}^{2}-n\right) C_{3}\right] \text {, } \\
& L_{56}=-n R\left[k_{2}^{2}\left(k_{1}^{2}-k_{2}^{2}\right) S_{2}+k_{3}^{2}\left(k_{1}^{2}-k_{3}^{2}\right) S_{3}\right] \text {, }
\end{aligned}
$$




$$
\begin{aligned}
& L_{61}=-\eta G_{r} R\left(k_{1}^{2} C_{1}+k_{2}^{2} C_{2}+k_{3}^{2} C_{3}\right), \\
& L_{62}=\frac{\eta F}{n} L_{26}, \\
& L_{63}=\xi L_{36}, \\
& L_{64}=L_{31} \\
& L_{65}=\frac{\eta}{n} L_{56}, \\
& L_{66}=R\left[\left(k_{2}^{2}-F\right)\left(k_{1}^{2}-k_{2}^{2}\right) C_{2}+\left(k_{3}^{2}-F\right)\left(k_{1}^{2}-k_{3}^{2}\right) C_{3}\right] .
\end{aligned}
$$

It is worth mentioning here that (2.32) have been used repeatedly in order to write the above entries in the simplest possible form. We will stress here that the above expression for the matrix exponential is a formal one. In the actual physical problem, the space is divided into two regions accordingly as $y \geq 0$ or $y<0$. Inside the region $0 \leq y \leq \infty$, the positive exponential terms, not bounded at infinity, must be suppressed. Thus, for $y \geq 0$, we should replace each $\sinh (k y)$ by $-(1 / 2) \exp (-k y)$ and each $\cosh (k y)$ by $(1 / 2) \exp (-k y)$. In the region $y \leq 0$, the negative exponentials are suppressed instead.

We will now proceed to obtain the solution of the problem for the region $y \geq 0$. The solution for the other region is obtained by replacing each $y$ by $-y$.

The formal solution of system (2.26) can be written in the form

$$
\bar{v}(y, s)=\exp (A(s) y)\left[\bar{v}(0, s)+\int_{0}^{y} \exp (-A(s) z) B(z, s) d z\right] .
$$

Evaluating the integral in (2.39) using the integral properties of the Dirac delta function, we obtain

$$
\bar{v}(y, s)=L(y, s)[\bar{v}(0, s)+H(s)],
$$

where

$$
H(s)=\frac{-Q_{0}\left(1+v_{o} s\right)}{2 s}\left[\begin{array}{c}
\frac{1}{2 k_{1}} \\
\frac{G_{r}\left[k_{1} k_{2} k_{3}+\xi\left(k_{1}+k_{2}+k_{3}\right)\right]}{2 k_{1} k_{2} k_{3}\left(k_{1}+k_{2}\right)\left(k_{1}+k_{3}\right)\left(k_{2}+k_{3}\right)} \\
0 \\
\frac{1}{2} \\
0 \\
-\eta G_{r} \\
\frac{\left.-\eta k_{1}+k_{2}\right)\left(k_{1}+k_{3}\right)\left(k_{2}\right)}{2\left(k_{1}\right)}
\end{array}\right] .
$$


Equation (2.40) expresses the solution of the problem in the Laplace transform domain for $y \geq 0$ in terms of the vector $H(s)$ representing the applied heat source and the vector $\bar{v}(0, s)$ representing the conditions at the plate $y=0$. To evaluate the components of this vector, we note first, due to the symmetry of the problem, that the velocity component and induced magnetic field component vanish at the plane source of heat, thus

$$
\begin{aligned}
& h(0, t)=0, \quad \text { or } \quad \bar{h}(0, s)=0, \\
& u(0, t)=0, \quad \text { or } \quad \bar{u}(0, s)=0 .
\end{aligned}
$$

Gauss's divergence theorem will now be used to obtain the thermal condition at the plane source. We consider a short cylinder of unit base, whose axis is perpendicular to the plane source of heat and whose bases lie on opposite sides of it. Taking the limit as the height of the cylinder tends to zero and noting that there is no heat flux through the lateral surface, we get

$$
q(0, t)=\frac{\mathcal{Q}_{o}}{2} H(t), \quad \text { or } \quad \bar{q}(0, s)=\frac{\mathcal{Q}_{o}}{2 s} .
$$

We will use the generalized Fourier's law of heat conduction in the nondimensional form [31], namely,

$$
q+v_{o} \frac{\partial q}{\partial t}=-\frac{\partial \theta}{\partial y}
$$

Taking the Laplace transform of both sides of this equation and using (2.43), we obtain

$$
\bar{\theta}^{\prime}(0, s)=\frac{-Q_{o}\left(1+v_{o} s\right)}{2 s} .
$$

Equation (2.42) and (2.45) give three components of the $\bar{v}(0, s)$. To obtain the remaining three components, we substitute $y=0$ on both sides of (2.40) getting a system of linear equations whose solution gives

$$
\begin{gathered}
\bar{\theta}(0, s)=\frac{-Q_{o}\left(1+v_{o} s\right)}{2 s k_{1}}, \\
\bar{u}^{\prime}(0, s)=\frac{w G_{r} Q_{0}\left(1+v_{o} s\right)}{2 s k_{1}\left(k_{1}+k_{2}\right)\left(k_{1}+k_{3}\right)\left(k_{2} k_{3}+\xi\right)}, \\
\bar{h}^{\prime}(0, s)=\frac{\eta G_{r} Q_{o}\left(1+v_{o} s\right)}{2 s k_{1}\left(k_{1}+k_{2}\right)\left(k_{1}+k_{3}\right)\left(k_{2} k_{3}+\xi\right)} .
\end{gathered}
$$


Inserting the values from (2.42) and (2.46) into the right-hand side of (2.40) and performing the necessary matrix operations, we obtain

$$
\begin{aligned}
\bar{\theta}(y, s)= & \frac{\mathcal{Q}_{o}\left(1+v_{o} s\right)}{2 s k_{1}} e^{-k_{1} y} \\
\bar{u}(y, s)= & \frac{-G_{r} Q_{o}\left(1+v_{o} s\right)}{2 s \beta \xi}\left[\left(k_{2}-k_{3}\right) A_{1} e^{-k_{1} y}+\left(k_{3}-k_{1}\right) A_{2} e^{-k_{2} y}+\left(k_{1}-k_{2}\right) A_{3} e^{-k_{3} y}\right] \\
\bar{h}(y, s)=\frac{-G_{r} \eta Q_{o}\left(1+v_{o} s\right)}{2 s \beta}[ & \left(k_{2}-k_{3}\right)\left(k_{2}+k_{3}-w\right) e^{-k_{1} y}+\left(k_{3}-k_{1}\right)\left(k_{1}+k_{3}-w\right) e^{-k_{2} y} \\
& \left.+\left(k_{1}-k_{2}\right)\left(k_{1}+k_{2}-w\right) e^{-k_{3} y}\right]
\end{aligned}
$$

where

$$
\begin{gathered}
w=k_{1} k_{2} k_{3}+\xi\left(k_{1}+k_{2}+k_{3}\right), \\
\beta=k_{1}\left(k_{1}^{2}-k_{2}^{2}\right)\left(k_{3}^{2}-k_{1}^{2}\right)\left(k_{2} a_{3}-k_{3} a_{2}\right), \\
A_{1}=w \xi\left(k_{1}-k_{2}\right)-a_{2}\left[a_{3}-\xi k_{3}\left(k_{1}+k_{3}\right)\right], \\
A_{2}=-a_{2}\left[a_{3}-\xi k_{3}\left(k_{1}+k_{3}\right)\right], \\
A_{3}=-w \xi\left(k_{2}-k_{3}\right)-a_{2}\left[a_{3}-\xi k_{3}\left(k_{1}+k_{3}\right)\right], \\
a_{2}=k_{2}^{2}-\xi, \quad a_{3}=k_{3}^{2}-\xi .
\end{gathered}
$$

Also, substituting from (2.49) into (2.21) the induced electric field is given by

$$
\begin{aligned}
\bar{E}(y, s)=\frac{G_{r} \eta Q_{o}\left(1+\tau_{o} s\right)}{2 \beta}[ & \left(k_{2}-k_{3}\right)\left(k_{2}+k_{3}-w\right) \frac{e^{-k_{1} y}}{k_{1}}+\left(k_{3}-k_{1}\right)\left(k_{1}+k_{3}-w\right) \frac{e^{-k_{2} y}}{k_{2}} \\
& \left.+\left(k_{1}-k_{2}\right)\left(k_{1}+k_{2}-w\right) \frac{e^{-k_{3} y}}{k_{3}}\right] .
\end{aligned}
$$

Equations (2.47)-(2.51) determine completely the state of the fluid for $y \geq 0$. We mention in passing that these equations give also the solution to a semispace problem with a plane source of heat on its boundary that constitutes a rigid base. As mentioned before, the solution for the whole space when $y<0$ is obtained from (2.47)-(2.51), by taking the symmetries under considerations.

We will show that the solution obtained above can be used as a set of building blocks from which the solutions to many interesting problems can be constructed. For future 
reference, we will write down the solution to the problem in the case when the source of heat is located in the plane $y=c$, instead of the plane $y=0$. In this case, we have

$$
\begin{aligned}
& \bar{\theta}(y, s)= \frac{\mathcal{Q}_{o}\left(1+v_{o} s\right)}{2 s k_{1}} e^{ \pm k_{1}(y-c)}, \\
& \bar{u}(y, s)=\frac{-G_{r} Q_{o}\left(1+v_{o} s\right)}{2 s \beta \xi}\left[\left(k_{2}-k_{3}\right) A_{1} e^{ \pm k_{1}(y-c)}+\left(k_{3}-k_{1}\right) A_{2} e^{ \pm k_{2}(y-c)}+\left(k_{1}-k_{2}\right) A_{3} e^{ \pm k_{3}(y-c)}\right], \\
& \bar{h}(y, s)=\frac{G_{r} \eta Q_{o}\left(1+v_{o} s\right)}{2 s \beta}\left[\left(k_{2}-k_{3}\right)\left(k_{2}+k_{3}-w\right) e^{ \pm k_{1}(y-c)}+\left(k_{3}-k_{1}\right)\left(k_{1}+k_{3}-w\right) e^{ \pm k_{2}(y-c)}\right. \\
&\left.+\left(k_{1}-k_{2}\right)\left(k_{1}+k_{2}-w\right) e^{ \pm k_{3}(y-c)}\right], \\
& \bar{E}(y, s)=\frac{G_{r} \eta Q_{o}\left(1+v_{o} s\right)}{2 \beta}\left[\left(k_{2}-k_{3}\right)\left(k_{2}+k_{3}-w\right) \frac{e^{ \pm k_{1}(y-c)}}{k_{1}}+\left(k_{3}-k_{1}\right)\left(k_{1}+k_{3}-w\right) \frac{e^{ \pm k_{2}(y-c)}}{k_{2}}\right. \\
&\left.\quad+\left(k_{1}-k_{2}\right)\left(k_{1}+k_{2}-w\right) \frac{e^{ \pm k_{3}(y-c)}}{k_{3}}\right],
\end{aligned}
$$

where the upper (plus) sign denotes the solution in the region $y \leq c$, while the lower (minus) sign denotes the solution in the region $y>c$.

\section{Applications}

We will now consider the problems of a semispace with a plane source of heat located inside the medium at the position $y=c$ and subject to the following boundary conditions.

(i) The shearing stress and the induced magnetic field are vanishing at the wall $(y=0)$,

$$
\begin{gathered}
\frac{\partial u(0, t)}{\partial y}=0, \quad \text { or } \quad \frac{\partial \bar{u}(0, s)}{\partial y}=0, \\
h(0, t)=0, \quad \text { or } \quad \bar{h}(0, s)=0 .
\end{gathered}
$$

(ii) The temperature is kept at a constant value $T_{\infty}$, which means that the temperature increment $\theta$ satisfies

$$
\theta(0, t)=0 \text { or } \bar{\theta}(0, s)=0 .
$$

This problem can be solved in a manner analogous to the outlined above though the calculations become quite messy. We will instead use the reflection method together with 
the solution obtained above for the whole space. This approach was proposed by Nowacki in the context of coupled thermoelasticity [31].

The boundary conditions of the problem can be satisfied by locating two heat sources in an infinite space, one positive at $y=c$ and the other negative of the same intensity at $y=-c$. The temperature increment $\theta$ is obtained as a superposition of the temperature for both plane distribution. Thus, $\theta=\theta_{1}+\theta_{2}$, where $\theta_{1}$ is the temperature due to the positive heat source, given by (2.52) and $\theta_{2}$ is the temperature due to the negative heat source and is obtained from (2.52) by replacing $c$ with $-c$, and noting that for all points of the semispace, we have $y+c>0$. Thus, $\theta_{2}$ is given by

$$
\theta_{2}(y, s)=\frac{\mathcal{Q}_{o}\left(1+v_{o} s\right)}{2 s k_{1}} e^{-k_{1}(y+c)}
$$

Combining (2.52) and (3.2), we obtain

$$
\begin{aligned}
& \bar{\theta}(y, s)=\frac{\mathcal{Q}_{o}\left(1+v_{o} s\right)}{2 s k_{1}} e^{-k_{1} y} \sinh k_{1} c \quad \text { for } y \geq c, \\
& \bar{\theta}(y, s)=\frac{\mathcal{Q}_{o}\left(1+v_{o} s\right)}{2 s k_{1}} e^{-k_{1} c} \sinh k_{1} y \quad \text { for } y \prec c .
\end{aligned}
$$

Clearly, this distribution satisfies the boundary condition (3.2). We turn now to the problem of finding the distributions velocity, the induced magnetic field, and the induced electric field. Unfortunately, the above procedure of superposition cannot be applied to these fields as in the temperature fields. We define the scalar stream function $\psi$ by the relation

$$
u=\frac{\partial \psi}{\partial y}
$$

By integration (2.53) and using (3.5), we obtain the stream function due to the positive heat source at the position $y=c$ as

$$
\psi=\frac{G_{r} Q_{o}\left(1+v_{o} s\right)}{2 s \beta \xi}\left[\left(k_{2}-k_{3}\right) A_{1} \frac{e^{ \pm k_{1}(y-c)}}{k_{1}}+\left(k_{3}-k_{1}\right) A_{2} \frac{e^{ \pm k_{2}(y-c)}}{k_{2}}+\left(k_{1}-k_{2}\right) A_{3} \frac{e^{ \pm k_{3}(y-c)}}{k_{3}}\right],
$$

where the upper sign is valid for the region $0 \leq y<c$ and the lower sign is valid for the region $y \geq 0$. Similarly, the stream function for the negative heat source at $y=-c$ is given by

$$
\psi=\frac{G_{r} Q_{o}\left(1+v_{o} s\right)}{2 s \beta \xi}\left[\left(k_{2}-k_{3}\right) A_{1} \frac{e^{-k_{1}(y+c)}}{k_{1}}+\left(k_{3}-k_{1}\right) A_{2} \frac{e^{-k_{2}(y+c)}}{k_{2}}+\left(k_{1}-k_{2}\right) A_{3} \frac{e^{-k_{3}(y+c)}}{k_{3}}\right] .
$$


Since $\psi$ is a scalar field, we can use superposition to obtain the stream function for the semispace problem as

$$
\psi=\left\{\begin{array}{ccc}
\frac{G_{r} Q_{o}\left(1+v_{o} s\right)}{s \beta \xi}[ & {\left[\left(k_{2}-k_{3}\right) A_{1} \frac{e^{-k_{1} y} \sinh k_{1} c}{k_{1}}+\left(k_{3}-k_{1}\right) A_{2} \frac{e^{-k_{2} y} \sinh k_{2} c}{k_{2}}\right.} & \\
& \left.+\left(k_{1}-k_{2}\right) A_{3} \frac{e^{-k_{3} y} \sinh k_{3} c}{k_{3}}\right] & \text { for } y \geq c, \\
\frac{G_{r} Q_{o}\left(1+v_{o} s\right)}{s \beta \xi}\left[\left(k_{2}-k_{3}\right) A_{1} \frac{e^{-k_{1} c} \sinh k_{1} y}{k_{1}}+\left(k_{3}-k_{1}\right) A_{2} \frac{e^{-k_{2} c} \sinh k_{2} y}{k_{2}}\right. & \\
& \left.+\left(k_{1}-k_{2}\right) A_{3} \frac{e^{-k_{3} c} \sinh k_{3} y}{k_{3}}\right] & \text { for } y \prec c .
\end{array}\right.
$$

Using (3.8) and (3.5), we obtain the velocity distribution

$$
\bar{u}=\left\{\begin{aligned}
\frac{-G_{r} Q_{o}\left(1+v_{o} s\right)}{s \beta \xi}[ & \left(k_{2}-k_{3}\right) A_{1} e^{-k_{1} y} \sinh k_{1} c \\
& +\left(k_{3}-k_{1}\right) A_{2} e^{-k_{2} y} \sinh k_{2} c \\
& \left.+\left(k_{1}-k_{2}\right) A_{3} e^{-k_{3} y} \sinh k_{3} c\right] \quad \text { for } y \geq c, \\
\frac{G_{r} Q_{o}\left(1+v_{o} s\right)}{s \beta \xi}[ & \left(k_{2}-k_{3}\right) A_{1} e^{-k_{1} c} \cosh k_{1} y \\
+ & \left(k_{3}-k_{1}\right) A_{2} e^{-k_{2} c} \cosh k_{2} y \\
+ & \left.\left(k_{1}-k_{2}\right) A_{3} e^{-k_{3} c} \cosh k_{3} y\right] \quad \text { for } y<c .
\end{aligned}\right.
$$

Differentiating (3.9) and using the resulting expressions together with (2.28), we obtain

$$
\bar{h}=\left\{\begin{aligned}
\frac{G_{r} \eta Q_{o}\left(1+v_{o} s\right)}{s \beta}[ & \left(k_{2}-k_{3}\right)\left(k_{2}+k_{3}-w\right) e^{-k_{1} y} \sinh k_{1} c \\
& +\left(k_{3}-k_{1}\right)\left(k_{1}+k_{3}-w\right) e^{-k_{2} y} \sinh k_{2} c \\
& \left.+\left(k_{1}-k_{2}\right)\left(k_{1}+k_{2}-w\right) e^{-k_{3} y} \sinh k_{3} c\right] \quad \text { for } y \geq c, \\
\frac{G_{r} \eta Q_{o}\left(1+v_{o} s\right)}{s \beta}[ & \left(k_{2}-k_{3}\right)\left(k_{2}+k_{3}-w\right) e^{-k_{1} c} \sinh k_{1} y \\
& +\left(k_{3}-k_{1}\right)\left(k_{1}+k_{3}-w\right) e^{-k_{2} c} \sinh k_{2} y \\
& \left.+\left(k_{1}-k_{2}\right)\left(k_{1}+k_{2}-w\right) e^{-k_{3} c} \sinh k_{3} y\right] \quad \text { for } y<c .
\end{aligned}\right.
$$




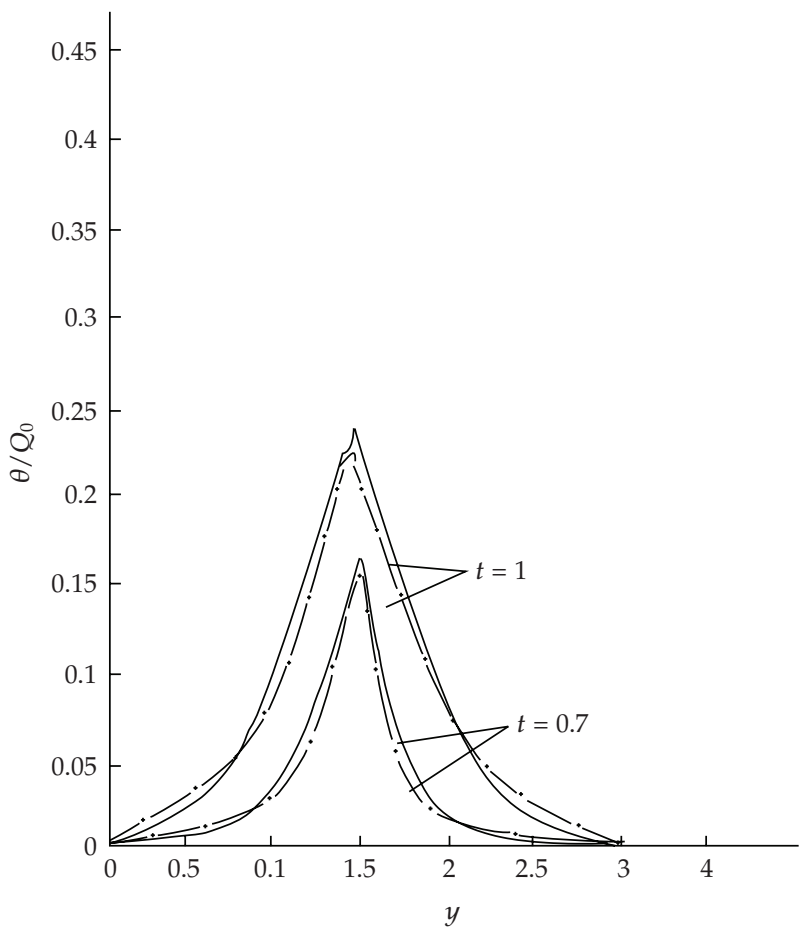

Figure 1: Variation of $\theta$ with $y,(-)$ for $v_{0}=0.03,(-\cdot)$ for $v_{0}=0.4$, and $t=0.7,1$ at $P_{r}=7, G_{r}=4, K=1.2$, $\alpha=0.3$, and $c=2$ representing onset stationary convection.

Substituting (3.10) and (2.21), we can get

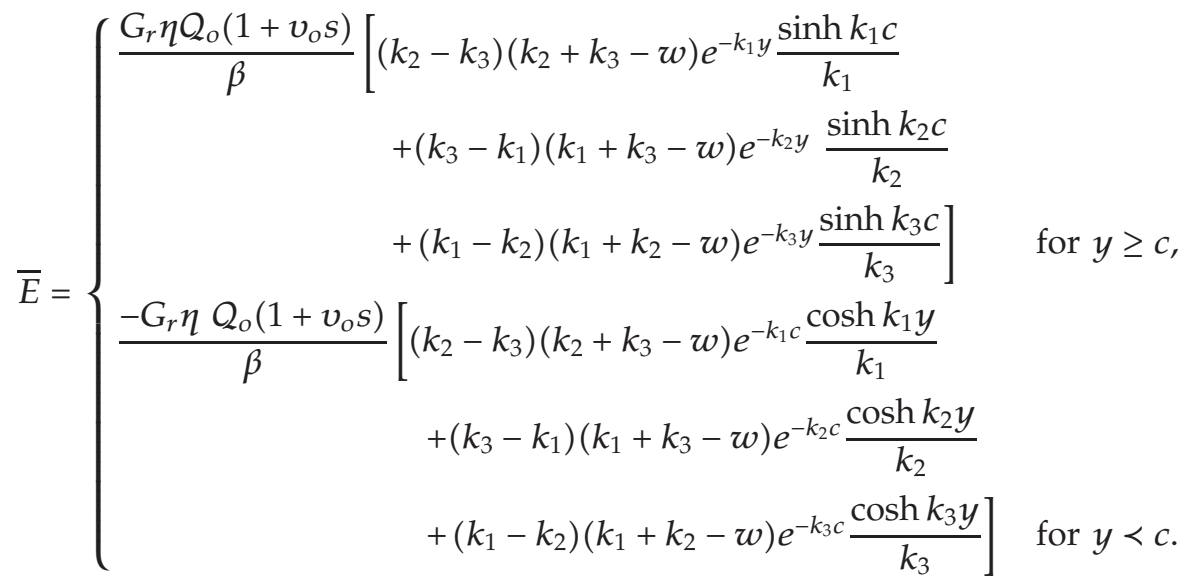

Clearly, $\partial \bar{u}(0, s) / \partial y=\bar{h}(0, s)=0$ in agreement with (3.1). 


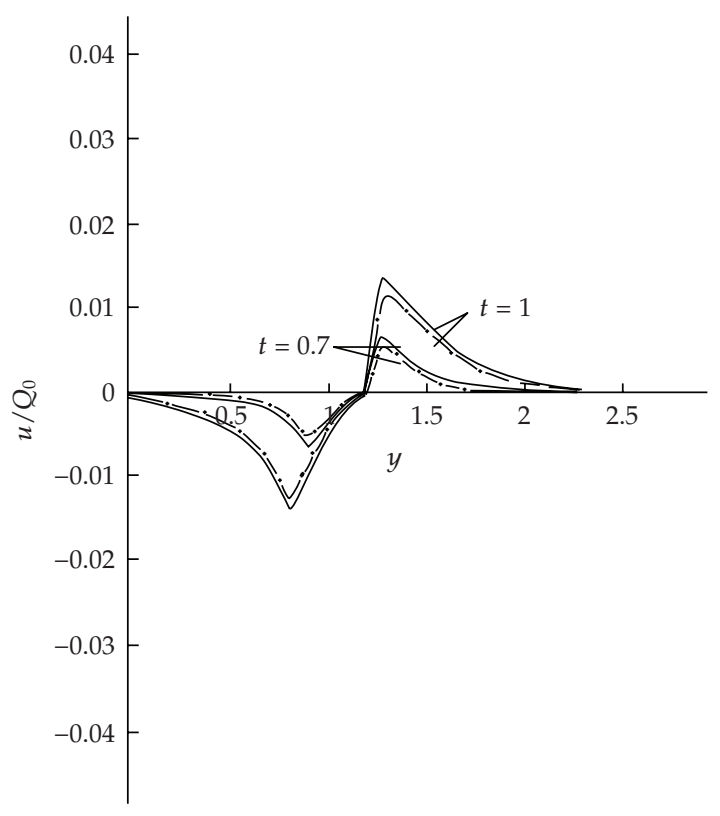

Figure 2: Variation of $u$ with $y_{,}(-)$for $v_{0}=0.03,(-\cdot)$ for $v_{0}=0.4$, and $t=0.7,1$ at $P_{r}=7, G_{r}=4, K=1.2$, $\alpha=0.3$, and $c=2$ representing onset stationary convection.

\section{Inversion of the Laplace Transform}

In order to invert the Laplace transforms in the above equations, we will use a numerical technique based on Fourier expansions of functions.

Let $\bar{g}(s)$ be the Laplace transform of a given function $g(t)$. The inversion formula of Laplace transforms states that

$$
g(t)=\frac{1}{2 \pi i} \int_{d-i \infty}^{d+i \infty} e^{s t} \bar{g}(s) d s
$$

where $d$ is an arbitrary positive constant greater than all the real parts of the singularities of $\bar{g}(s)$. Taking $s=d+i y$, we get

$$
g(t)=\frac{e^{d t}}{2 \pi} \int_{-\infty}^{\infty} e^{i t y} \bar{g}(d+i y) d y
$$

This integral can be approximated by

$$
g(t)=\frac{e^{d t}}{2 \pi} \sum_{k=-\infty}^{\infty} e^{i k t \Delta y} \bar{g}(d+i k \Delta y) \Delta y
$$




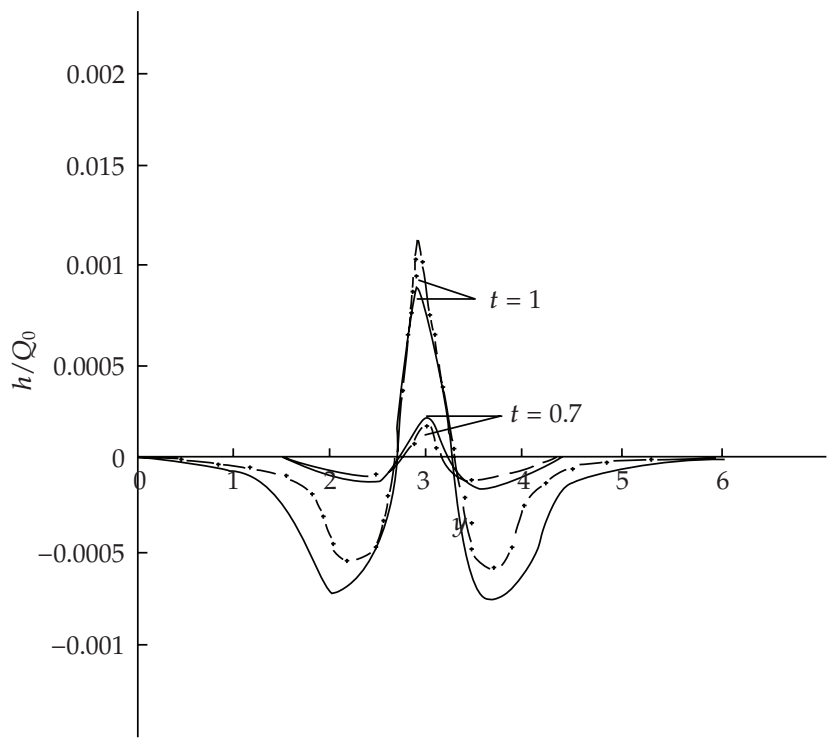

Figure 3: Variation of $h$ with $y,(-)$ for $v_{0}=0.03,(-\cdot)$ for $v_{0}=0.4$, and $t=0.7,1$ at $P_{r}=7, G_{r}=4, K=1.2$, $\alpha=0.3$, and $c=2$ representing onset stationary convection.

Taking $\Delta y=\pi / t_{1}$, we obtain

$$
g(t)=\frac{e^{d t}}{t_{1}}\left[\frac{1}{2} \bar{g}(d)+\operatorname{Re}\left(\sum_{k=1}^{\infty} e^{i k \pi t / t_{1}} \bar{g}\left(d+\frac{i k \pi}{t_{1}}\right)\right)\right] .
$$

For numerical purposes, this is approximated by the function

$$
g_{N}(t)=\frac{e^{d t}}{t_{1}}\left[\frac{1}{2} \bar{g}(d)+\operatorname{Re}\left(\sum_{k=1}^{N} e^{i k \pi t / t_{1}} \bar{g}\left(d+\frac{i k \pi}{t_{1}}\right)\right)\right]
$$

where $N$ is a sufficiently large integer chosen such that

$$
\frac{e^{d t}}{t_{1}} \operatorname{Re}\left[e^{i N \pi\left(t / t_{1}\right)} \bar{g}\left(d+\frac{i N \pi}{t_{1}}\right)\right] \prec \eta,
$$

where $\eta$ is a preselected small positive number that corresponds to the degree of accuracy to be achieved, Formula (3.11) is the numerical inversion formula valid for $0 \leq t \leq 2 t_{1}$ [22]. In particular, we choose $t=t_{1}$, getting

$$
g_{N}(t)=\frac{e^{d t}}{t}\left[\frac{1}{2} \bar{g}(d)+\operatorname{Re}\left(\sum_{k=1}^{N}(-1)^{k} \bar{g}\left(d+\frac{i k \pi}{t}\right)\right)\right] .
$$




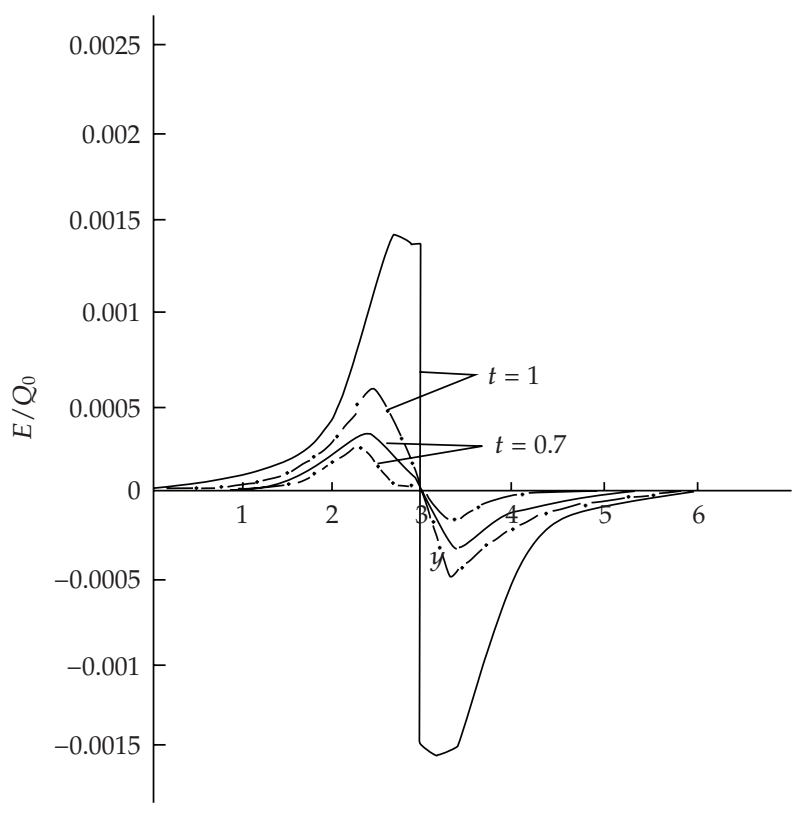

Figure 4: Variation of $E$ with $y,(-)$ for $v_{0}=0.03,(-\cdot)$ for $v_{0}=0.4$, and $t=0.7,1$ at $P_{r}=7, G_{r}=4, K=1.2$, $\alpha=0.3$, and $c=2$ representing onset stationary convection.

\section{Numerical Results}

The constants of the problem were taken as $\varepsilon_{O}=0.003, \alpha=0.3, G_{r}=4, K=1.2$, and $c=2$. All constants are given in SI units. The computations were carried out for the two different values of time, namely, $t=0.7$ and 1 . The functions $\theta, u, h$, and $E$ are evaluated. The results are shown in Figures 1, 2, 3, and 4. In these figures, solid lines represent the solution corresponding to using the generalized Fourier equation of heat conduction $\left(v_{o}=0.4\right)$, while dashed lines represent the solution corresponding to using the classical Fourier heat equation $\left(v_{o}=0.03\right)$.

The important phenomenon observed in all computations is that the solution of any of the considered functions vanishes identically outside a bounded region of space surrounding the heat source at a distance from it equal to $x^{*}(t)$, and say that $x^{*}(t)$ is a particular value of $y$ depending only on the choice of $t$ and is the location of the wave front. This demonstrates clearly the difference between the solution corresponding to using classical Fourier heat equation $\left(v_{o}=0.03\right)$ and to using the non-Fourier case $\left(v_{o}=0.4\right)$. In the first and older theory, the waves propagate with infinite speeds, so the value of any of the functions is not identically zero (though it may be very small) for any large value of $y$. In the non-Fourier theory, the response to the thermal and mechanical effects does not reach infinity instantaneously but remains in a bounded region of space given by $0 \prec y \prec y^{*}(t)$ for the semispace problem and by $\operatorname{Min}\left(0, y^{*}(t)-c\right) \prec y<y+y^{*}(t)$ for the whole space problem.

We notice that results for all functions considered in the semispace problem when the relaxation time is appeared in heat equation are distinctly different from those when the relaxation time disappeared.

We also notice that for small values of time, the solution is localized in a finite region near the plane of heat sources. This region grows with increasing time until it fills the whole boundary-layer region. 


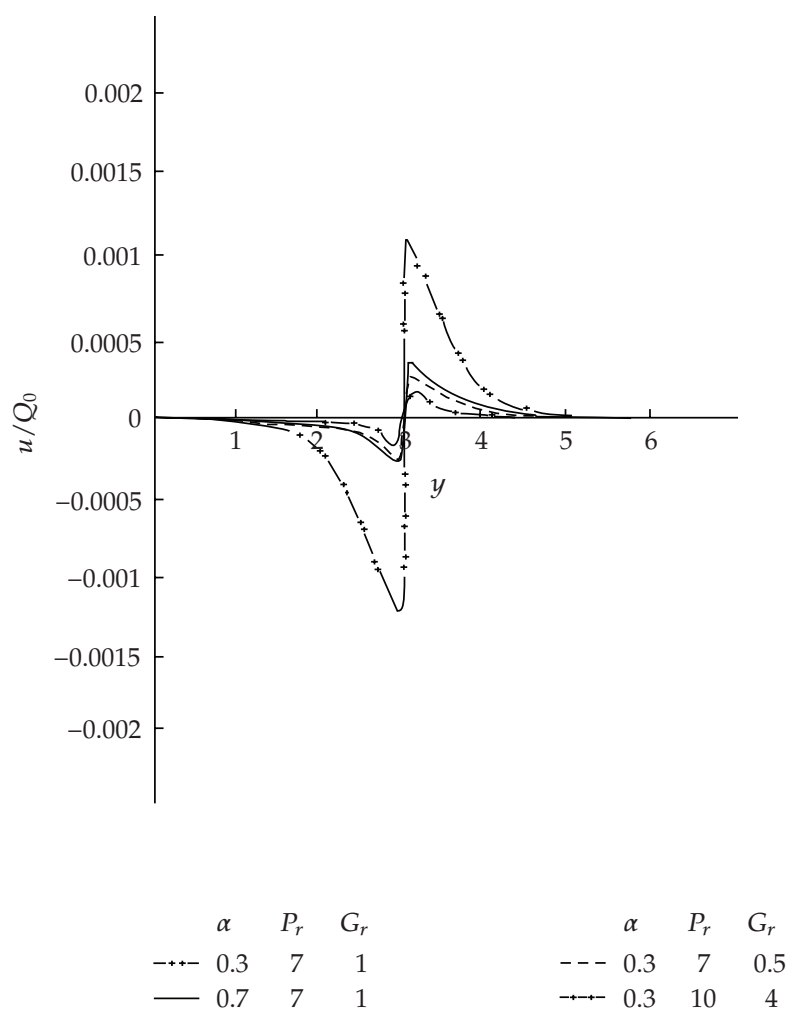

Figure 5: Velocity distribution $u$ with $y$, for different values of $\alpha, P_{r}$, and $G_{r}$, with $t=0.7, v_{0}=0.4, K=1$, and $c=2$ representing onset stationary convection.

Also, we observe from Figure 5. that the effect of heating by free convention currents when $G_{r}>0$. In this case, it is noticed that as Alfven velocity $\alpha$ increases, the velocity is found to decrease. This is mainly due to the fact that the effect of the magnetic field corresponds to a term signifying a positive force that tends to decelerate the fluid particles. Also, it is noticed that the velocity increases as $G_{r}$ increases, while it decreases when the Prandtl number $p_{r}$ increases.

We notice that results for the temperature distribution when the relaxation time is appears in the heat equation are distinctly different from those when the relaxation time is not mentioned in the heat equation. This is due to the fact that thermal waves in the Fourier theory of heat equation travel with an infinite speed of propagation as opposed to finite speed in the non-Fourier case. We also notice that for small values of time, the solution is localized in a finite region near the plane surface. This region grows with increasing time until it fills the whole boundary-layer region. As time $t$ increases, results for both theories of heat equation almost coincide which is expected, since magnetohydrodynamic free convection flow effects are short lived. At all values of time, the velocity distributions for both theories coincide.

The effect of the relaxation time on the variation temperature and velocity distribution for this problem is shown in Figures 1 and 2, respectively.

The values of Grashof number $G_{r}$ have been chosen as they are interesting from physical point of view. 


\section{Concluding Remarks}

Many metallic materials are manufactured after they have been redefined sufficiently in the molten state. Therefore, it is a central problem in metallurgical chemistry to study the free convection effects on conducting fluid metal. For instance, liquid sodium $\mathrm{Na}\left(100^{\circ} \mathrm{C}\right)$ exhibit very small electrical resistivity.

The effects of Grashof number, Alfven velocity, Prandtl number, and relaxation time on the oneset temperature and velocity distribution are discussed. A discussion is provided for the effects of heating on a viscous conducting fluid is given.

The importance of state space analysis is recognized in fields where the time behavior of any physical process is of interest.

The state-space approach is more general than the classical Laplace and Fourier transform techniques. Consequently, state space is applicable to all systems that can be analyzed by integral transforms in time and is applicable to many systems for which transform theory breaks down [32].

Owing to the complicated nature of the governing equations for the unsteady magnetohydrodynamic with gradient pressure flow, few attempts have been made to solve problems in this field. These attempts utilized approximate methods valid for only a specific range of some parameters.

In this work, the method of direct integration by means of the matrix exponential, which is a standard approach in modern control theory and developed in detail in many texts such as Ogata [33], and Ezzat et al. [34, 35], is introduced in the field of magnetohydrodynamics and applied to two specific problems in which the temperature and velocity are coupled. This method gives exact solutions in the Laplace transform domain without any assumed restrictions on either the applied magnetic field or the velocity, temperature distributions, and viscoelastic parameter.

The method used in the present work is applicable to a wide range of problems. It can be applied to problems which are described by the linearized Navier-Stokes equations. The same approach was used quite successfully in dealing with problems in thermoelasticity theory $[14,15,19,34,35]$.

\section{Nomenclature}

$t$ : Time

$x, y$ : Coordinates system

$v$ : $=(u, 0,0)$ velocity vector of the fluid

$T: \quad$ Temperature distribution

$T_{o}$ : Temperature of the plate

$T_{\infty}$ : Temperature of the fluid away from the plate

$p_{r}$ : Prandtl number

$G_{r}$ : Grashof number

$c_{p}$ : Specific heat at constant pressure

g: Acceleration due to gravity

$\rho: \quad$ Density

$\lambda$ : Thermal diffusivity

$h$ : Induced magnetic field

$Q_{0}$ : Constant

Q: The strength of the applied heat source 


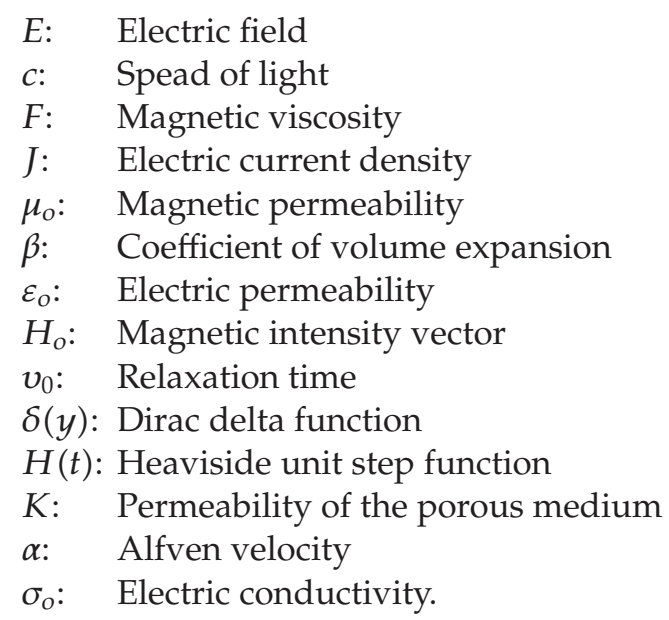

\section{Acknowledgment}

The author is very grateful to the referees for their helpful suggestions which improved the paper.

\section{References}

[1] A. A. Samaan, "Oscillating in a circular pipe with adverse pressure gradient," International Journal of Dynamics of Fluids, vol. 5, pp. 119-127, 2009.

[2] A. A. Samaan, "Analytical and numerical methods for the momentum, species concentration and energy equations of a viscous incompressible fluid along a vertical plate," Nuovo Cimento della Società Italiana di Fisica B, vol. 125, no. 4, pp. 439-455, 2010.

[3] A. J. Chamkha, "MHD flow of a uniformly streched vertical permeable surface in the presence of heat generation/absorption and a chemical reaction," International Communications in Heat and Mass Transfer, vol. 30, no. 3, pp. 413-422, 2003.

[4] A. Ishak, R. Nazar, and I. Pop, "Unsteady mixed convection boundary layer flow due to a stretching vertical surface," The Arabian Journal for Science and Engineering B, vol. 31, no. 2, pp. 165-182, 2006.

[5] S. J. Liao and I. Pop, "Explicit analytic solution for similarity boundary layer equations," International Journal of Heat and Mass Transfer, vol. 47, no. 1, pp. 75-85, 2004.

[6] R. Nazar, N. Amin, and I. Pop, “Unsteady boundary layer flow due to a stretching surface in a rotating fluid," Mechanics Research Communications, vol. 31, no. 1, pp. 121-128, 2004.

[7] C. Y. Wang, "Stagnation flow towards a shrinking sheet," International Journal of Non-Linear Mechanics, vol. 43, no. 5, pp. 377-382, 2008.

[8] K. R. Singh, V. K. Agrawal, and A. Singh, "Heat transfer in the flow of a non-Newtonian second-order fluid between two enclosed counter torsionally oscillating DISC," International Journal of Dynamics of Fluids, vol. 4, pp. 145-157, 2008.

[9] A. A. Samaan, "Heat and mass transfer over an accelerating surface with heat source in presence of Suction," International Journal of Theoretical and Applied Mechanics, vol. 3, pp. 195-206, 2008.

[10] R. Muhammad, K. Fakhar, and A. Farooq, "Unsteady magneto-fluid-dynamics fluid and heat flow," International Journal of Dynamics of Fluids, vol. 4, pp. 1-12, 2008.

[11] A. A. Samaan, "Heat and mass transfer over an accelerating surface with heat source in presence of magnetic field," International Journal of Theoretical and Applied Mechanics, vol. 4, pp. 279-291, 2009.

[12] A. A. Samaan, "The effects of variable viscosity and thermal diffusivity on the steady flow in the presence of the magnetic field," Nuovo Cimento della Societa Italiana di Fisica B, vol. 124, no. 9, pp. 931-946, 2009. 
[13] A. A. Mostafa, "A note on variable viscosity and chemical reaction effect on mixed convection heat and mass transfer along a seme-infinite vertical plate," Mathematical Problems in Engineering, vol. 10, pp. 1-7, 2007.

[14] A. A. Samaan, "State space formulation for magnetohydrodynamic free convection flow with two relaxation times," Applied Mathematics and Computation, vol. 152, no. 2, pp. 299-321, 2004.

[15] M. A. Ezzat, M. Zakaria, A. A. Samaan, and A. Abd Elbary, "Free convection effective perfectly conducting couple stress fluid," Journal of Technical Physics, vol. 47, pp. 5-30, 2006.

[16] T. Hayat, H. Mambili-Mamboundou, and F. M. Mahomed, "Unsteady solutions in a third-grade fluid filling the porous space," Mathematical Problems in Engineering, vol. 2008, Article ID 139560, 13 pages, 2008.

[17] M. M. Rashidi and M. Keimanesh, “Using differential transform method and padé approximant for solving mhd flow in a laminar liquid film from a horizontal stretching surface," Mathematical Problems in Engineering, vol. 2010, Article ID 491319, 2010.

[18] M. A. Ezzat and M. Z. Abd-Elaal, "Free convection effects on a viscoelastic boundary layer flow with one relaxation time through a porous medium," Journal of the Franklin Institute B, vol. 334, no. 4, pp. 685-706, 1997.

[19] M. A. Ezzat, A. S. El-Karamany, and A. A. Samaan, "The dependence of the modulus of elasticity on reference temperature in generalized thermoelasticity with thermal relaxation," Applied Mathematics and Computation, vol. 147, no. 1, pp. 169-189, 2004.

[20] C. Cattaneo, "On the conduction of heat," Comptes Rendus de l'Académie des Sciences, vol. 4, pp. 247431, 1958.

[21] P. Vernotte, "Les paradoxes de la théorie continue de l'équation de la chaleur," Comptes Rendus de l'Académie des Sciences, vol. 246, pp. 3154-3155, 1958.

[22] R. B. Hetnarski and J. Ignaczak, "Generalized thermoelasticity," Journal of Thermal Stresses, vol. 22 , no. 4-5, pp. 451-476, 1999.

[23] M. A. Ezzat, "State space approach to unsteady two-dimensional free convection flow through a porous medium," Canadian Journal of Physics, vol. 72, no. 5-6, pp. 311-317, 1994.

[24] M. A. Ezzat, "Fundamental solution in thermoelasticity with two relaxation times for cylindrical regions," International Journal of Engineering Science, vol. 33, no. 14, pp. 2011-2020, 1995.

[25] G. Honig and U. Hirdes, "A method for the numerical inversion of Laplace transforms," Journal of Computational and Applied Mathematics, vol. 10, no. 1, pp. 113-132, 1984.

[26] A. H. Nayfeh and S. Nemat-Nasser, "Electromagneto- thermoelastic plane waves in solids," Journal of Applied Mechanics E, vol. 39, pp. 108-113, 1972.

[27] J. P. Holman, Heat Transfer, Mc Graw-Hill/Kogahusha, Tokyo, Japan, 1976.

[28] M. A. Ezzat, "Free convection effects on perfectly conducting fluid," International Journal of Engineering Science, vol. 39, no. 7, pp. 799-819, 2001.

[29] D. W. Beard and K. Walters, "Elastico-viscous boundary-layer flows. I. Two-dimensional flow near a stagnation point," Proceedings of the Cambridge Philosophical Society, vol. 60, pp. 667-671, 1964.

[30] J. P. Goedbloed and S. Poedts, Principles of Magnetohydrodynamics, Cambridge University Press, Cambridge, UK, 2004.

[31] W. Nowacki, "Some dynamic problems of thermoelasticity," Archiwum Mechaniki Stosowanej, vol. 11, pp. 259-283, 1959.

[32] D. Wibery, Theory and Problems of State Space and Linear System, Schaum's Outline Series in Engineering, McGraw-Hill, New York, NY, USA, 1971.

[33] K. Ogata, State Space Analysis Control System, chapter 6, Prentice-Hall, Englewood Cliffs, NJ, USA, 1967.

[34] M. A. Ezzat, A. S. El-Karamany, and A. A. Samaan, "State-space formulation to generalized thermoviscoelasticity with thermal relaxation," Journal of Thermal Stresses, vol. 24, no. 9, pp. 823-846, 2001.

[35] M. A. Ezzat, M. I. Othman, and A. A. Smaan, "State space approach to two-dimensional electromagneto-thermoelastic problem with two relaxation times," International Journal of Engineering Science, vol. 39, no. 12, pp. 1383-1404, 2001. 


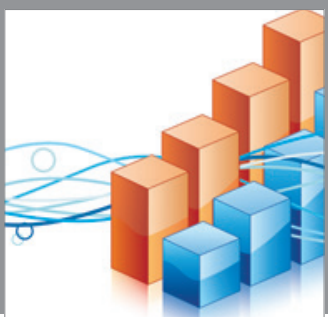

Advances in

Operations Research

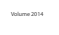

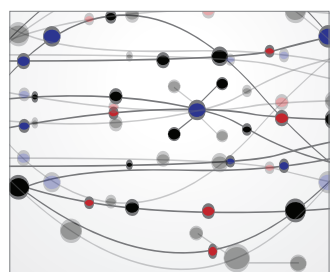

\section{The Scientific} World Journal
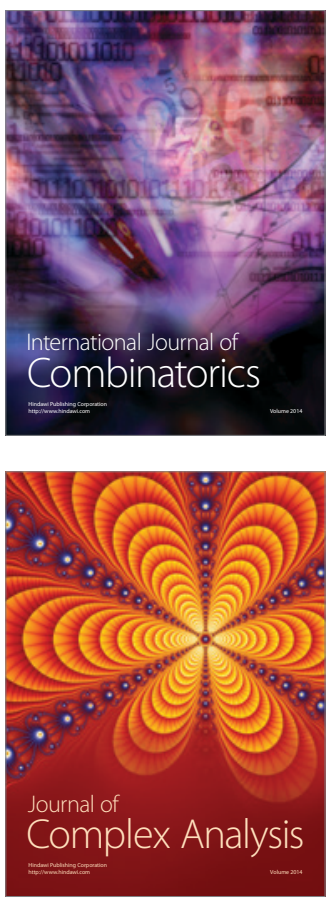

International Journal of

Mathematics and

Mathematical

Sciences
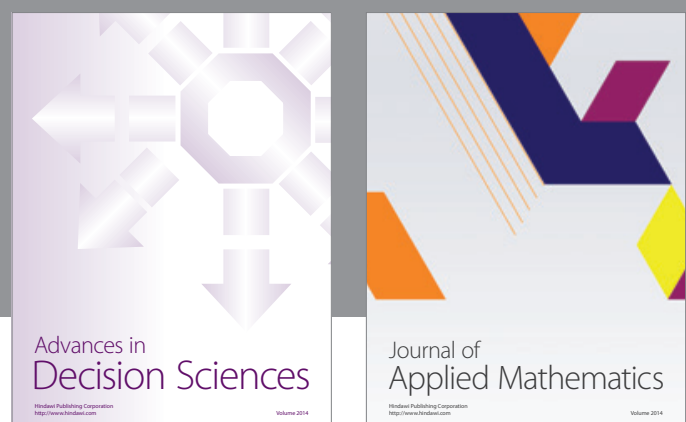

Journal of

Applied Mathematics
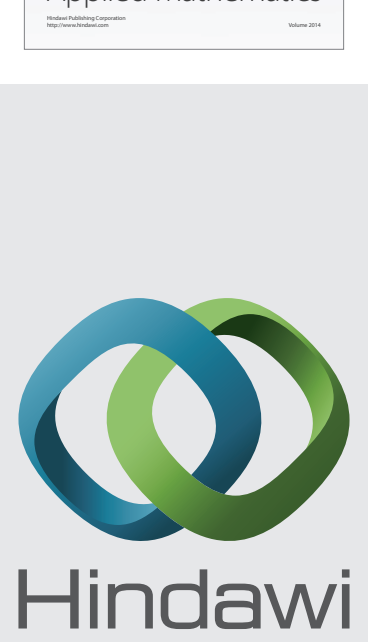

Submit your manuscripts at http://www.hindawi.com
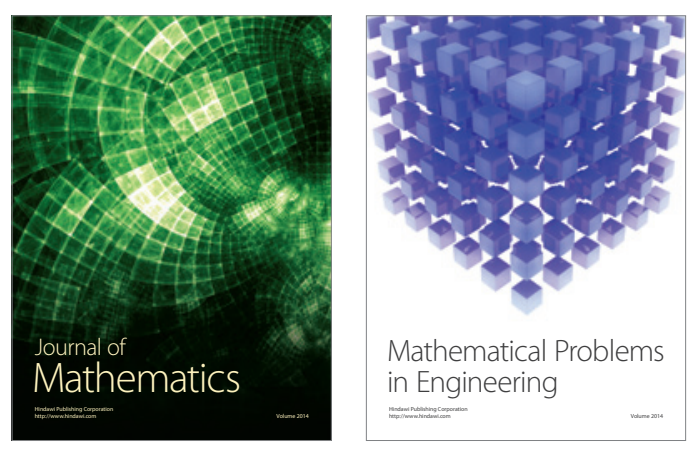

Mathematical Problems in Engineering
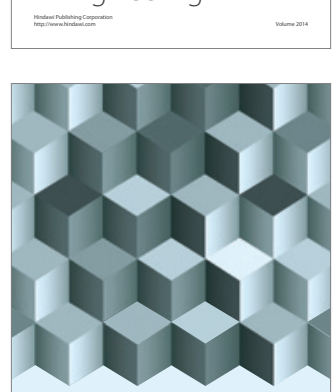

Journal of

Function Spaces
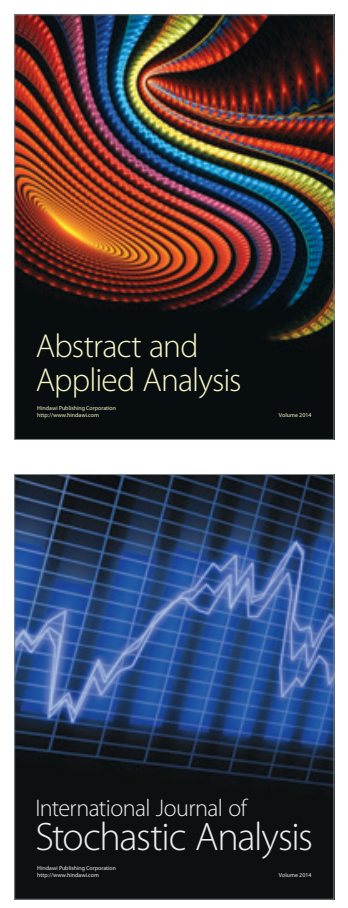

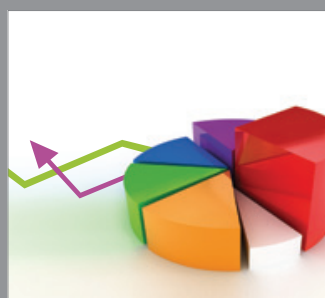

ournal of

Probability and Statistics

Promensencen
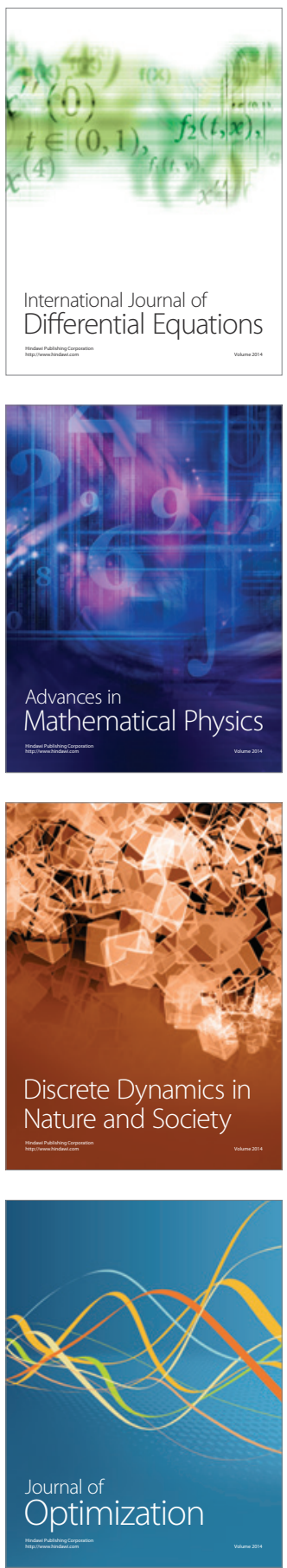